\title{
Practical X-ray Ghost Imaging
}

Andrew M. Kingston ${ }^{1,2 *}$, Glenn R. Myers ${ }^{1,2}$, Margie P. Olbinado ${ }^{3}$, Alexander Rack ${ }^{3}$, Daniele Pelliccia ${ }^{4}$ and David M. Paganin ${ }^{5}$

1. Dept. Applied Math., RSPE, The Australian National University, Canberra, ACT 2601, Australia

2. CTLab: National Laboratory for Micro-CT, Advanced Imaging Precinct, The Australian National University, Canberra, ACT 2601, Australia

3. ESRF: The European Synchrotron, 38043 Grenoble, France

4. Instruments \& Data Tools Pty Ltd, Victoria 3178, Australia

5. School of Physics and Astronomy, Monash University, Victoria 3800, Australia

* Corresponding author, andrew.kingston@anu.edu.au

Consider the proverbial monkey generating random text at a typewriter. Rather than waiting the vast time required for the monkey to type a page of Shakespeare's Macbeth, suppose that one only waits the time needed to type a thousand pages of random text. Can one view these thousand pages of random text as a basis over which to decompose an arbitrary text? Stated differently, can one form a linear combination of random texts, to synthesise a particular desired text such as a page from Macbeth [1]?

Replace the set of random monkey-generated texts with random two-dimensional speckled intensity fields, and consider this ensemble of random speckle fields as a basis from which one can synthesise arbitrary signals such as the real transmission function of a thin object. This is the key concept of ghost imaging. The random-speckle basis may be generated using e.g. shot noise from individual electron bunches in an X-ray synchrotron [2], or scattering of X-rays from a spatially random mask [3, 4]. A series of transverse translations of the speckle mask results in an ensemble of $M$ linearly independent speckle fields $I_{j}(x, y)$ that serve as the basis from which the X-ray ghost image is synthesised.

Figure 1 shows our experimental setup for practical X-ray ghost imaging in two spatial dimensions. Xrays (pink beam with mean energy $26.3 \mathrm{keV}$ ) at beamline ID19 of the European Synchrotron illuminate a $1 \mathrm{~cm}$ thick slab of glass powder, which generates a spatially random speckle field that is measured as the "reference image" over the surface of a position-sensitive detector $5.8 \mathrm{~m}$ downstream of the mask (speckle contrast 14\%). A Si beam splitter in Laue geometry generates a diffracted beam (220 reflection) passing through the sample (a lead sheet in which three circular holes have been drilled), before striking the surface of the detector. This diffracted beam is integrated over the area denoted "synthesised bucket", to give a so-called "bucket signal" $B_{j}$. Only photons that do not pass through the object are registered by a position-sensitive detector. The reference speckles measured by the detector are taken to be the same as the speckles illuminating the object when each bucket signal is acquired.

In its simplest form, the intensity transmission function $T(x, y)$ of the object can be reconstructed using the intensity-intensity correlation $T(x, y) \approx(1 / M) \sum_{j=1}^{M} \widetilde{B}_{j} I_{j}(x, y)$, where $\widetilde{B}_{j}=B_{j}-\bar{B}$ and $\bar{B}$ is the average of the bucket values $B_{j}[5,6]$. Thus $T(x, y)$ is expressed as a linear combination of the randomspeckle basis elements. This reconstruction (see Fig. 2(b)) may be refined using Landweber iteration [7], using $M=5000$ bucket measurements, yielding the reconstruction in Fig. 2(c). 


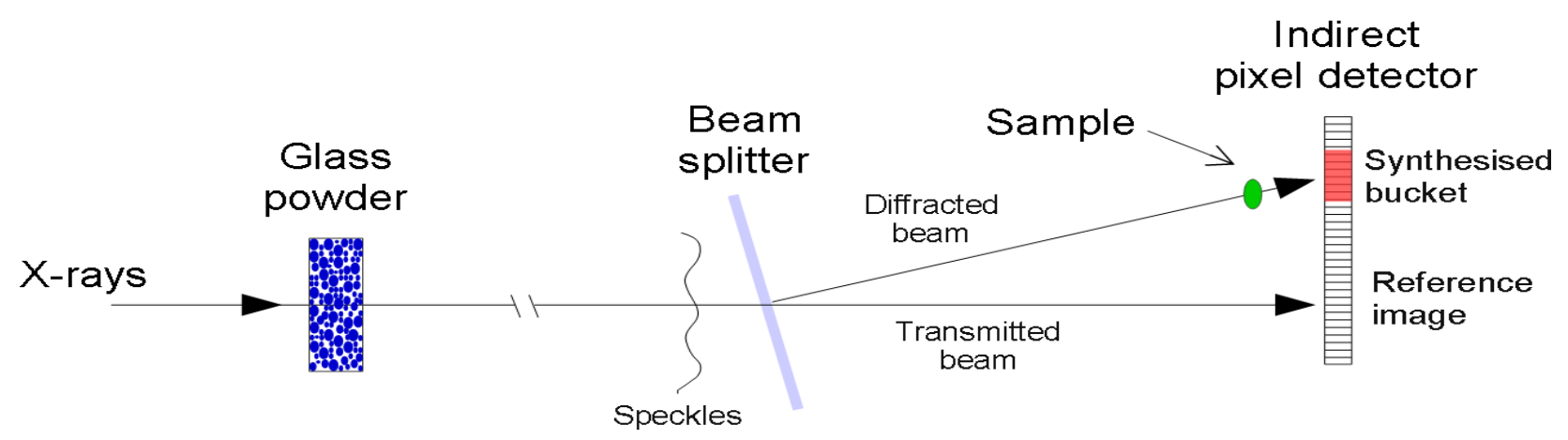

Figure. 1. Schematic diagram of the experimental setup.
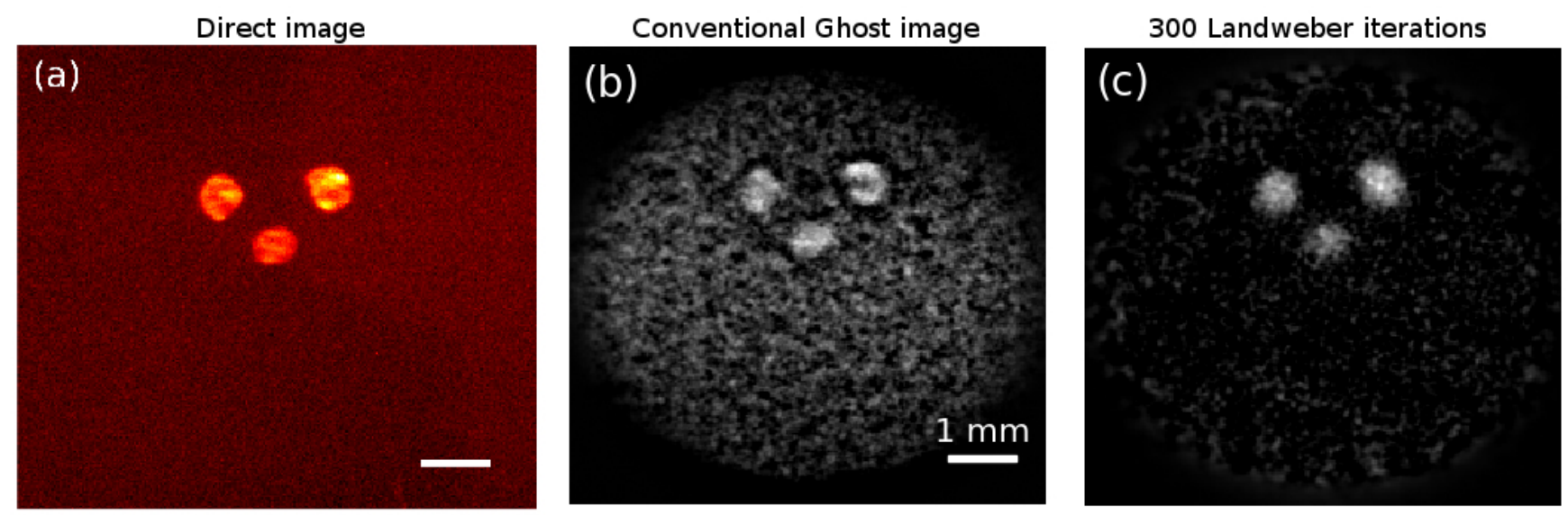

Figure. 2. (a) Direct image of three-hole lead stencil; (b) conventional ghost imaging reconstruction using $M=5000$; (c) reconstruction refined using 300 Landweber iterations with smoothing prior.

The ghost-image resolution is limited only by the speckle size (width of auto-covariance of the specklemap ensemble [8]). More precisely, the resolution is limited by the smallest spatial frequencies present to a non-negligible degree in the reference speckle images. The method may be viewed as a form of scanning-probe microscopy for which the probe covers the entire sample; again, resolution is governed by the speckle size, rather than the probe size. From another perspective, the method may also be viewed as a form of parallel intensity interferometer. Compressive sensing can improve efficiency [9].

[1] D Ceddia and DM Paganin, arXiv (2018) 1802.04258.

[2] D Pelliccia, A Rack, M Scheel, V Cantelli, and DM Paganin, Phys. Rev. Lett. 117 (2016) 113902.

[3] H Yu, R Lu, S Han, H Xie, G Du, T Xiao, and D Zhu, Phys. Rev. Lett. 117 (2016) 113901.

[4] A Schori and S Schwartz, Opt. Express 25 (2017) 14822.

[5] Y Bromberg, O Katz, and Y Silberberg, Phys. Rev. A 79 (2009) 053840.

[6] O Katz, Y Bromberg, and Y Silberberg, Appl. Phys. Lett. 95 (2009) 131110.

[7] H Huang, C Zhou, T Tian, D Liu, and L Song, Opt. Commun. 412 (2018) 60.

[8] F Ferri, D Magatti, LA Lugiato, and A Gatti, Phys. Rev. Lett. 104 (2010) 253603.

[9] We thank S Bérujon and E Brun for loaning us their speckle masks. We acknowledge discussions with TE Gureyev, ID Svalbe, and D Ceddia. AMK and GRM acknowledge the financial support of the Australian Research Council and FEI-Thermo Fisher Scientific through Linkage Project LP150101040. We thank the ESRF for granting beamtime (proposal MI-1283). 